\title{
Effect of defect accumulation on ion-beam damage morphology by electronic excitation in lithium niobate: A MonteCarlo approach
}

\author{
A. Rivera ${ }^{a}$, M.L. Crespillo ${ }^{b}$, J. Olivares ${ }^{\text {b.c }}$, G. García ${ }^{d}$, F. Agulló-López ${ }^{\text {b.e.* }}$ \\ a instituto de Microelectrónica de Madrid, (CNM-CSIC), isaac Newton 8, E-28760 Tres Cantos, Spain \\ -Centro de Microanálisis de Materiales (CMAM), Universidad Autónoma de Madrid (UAM), Contoblanco, E-28049 Madrid, Spain \\ "Instituto de Óptica, Consejo Superior de Investigaciones Científicas (CSIC). C/Serrano 121, E-28006 Madrid, Spain \\ ¿Laboratory of Synchrotron Light (CELLS-ALBA), 08290, Cerdanyola del Vallès, Barcelona, Spain \\ EDepartamento de Fisica de Materiales, Universidad Axtónoma de Madijd (UAM), Cantobianco, E-28049 Madrid, Spain
}

Keywords:

Damage

Excitons

lon-beams

Thermal spike

Rutherford backscattering channeling

Amorphization threshold

Montecarlo simulations

\begin{abstract}
A B S T R A C T
We present a MonteCarlo approach to the non-radiative exciton-decay model recently proposed to describe ion-beam damage in $\mathrm{LiNbO}_{3}$ produced in the electronic excitation regime. It takes into account the statistical (random) spatial distribution of ion impacts on the crystal surface. The MonteCarlo approach is necessary to simulate the evolution of the damage morphology with irradiation fluence from the single track regime to the overlapping track regime. A detailed comparison between the morphologies found for sub-threshold and above threshold irradiations is presented. Moreover, a good representation of the Avrami's type kinetics for amorphization has been achieved and it is in fair accordance with experiment. For moderate fluences where homogeneous amorphous layers are generated, the new approach predicts that the amorphous and crystalline layers are separated by a diffuse (thick) boundary that includes a mixed amorphous-crystalline composition.
\end{abstract}

\section{Introduction}

It is being increasingly recognized that the electronic stopping power $\left(S_{\mathrm{e}}\right)$ component of the energy losses of jon-beams impinging on dielectric materials may cause a largely enhanced damage in comparison with that associated to nuclear collisions, as illustrated for $\mathrm{LiNbO}_{3}$ [1]. This (electronic) damage is particularly relevant for high-energy medium-mass ions for which $S_{\mathrm{e}}$ is overwhelmingly dominant on the nuclear stopping power $\left(S_{\mathrm{n}}\right)$. Moreover, the electronic damage in $\mathrm{LiNbO}_{3}$ and possibly other dielectric $\left(\mathrm{KGW}, \mathrm{TiO}_{2}\right)$ and semiconductor ( $\ln \mathrm{P}, \ldots$ ) crystals presents peculiar characteristics [1-8] that are very different from those corresponding to nuclear collision damage, namely, the abrupt non-linear damage generation as a function of stopping power, the abrupt appearance of amorphous single tracks (common in many insulators) when $S_{e}$ exceeds a threshold value and the cumulative character of damage generation. On the other hand, the spatial distribution of damage is quite different for both mechanisms $[1,4,9]$. Electronic damage may occur over most range of the ion and becomes almost negligible at low ion energies $(<100 \mathrm{keV})$, whereas nuclear damage con- centrates at these low energies, at which the ion is close to its stop and implantation [10-12]. Actually, the nature of electronic damage and its operative mechanisms are still not sufficiently known. This is at variance with nuclear collision damage, where advanced simulation codes [10] are available and provide a satisfactory quantitative description of the amount and morphology of the damage regions.

For the electronic damage a phenomenological non-radiative exciton model has been recently developed $[13,14]$. It provides a quantitative estimation for the rate of irradiation-induced defects. The model results from the synergy between the spikes generated by irradiation, i.e., the thermal spike (due to the deposited energy converted into heat) and the exciton spike (due to deposited energy stored in electron-hole pairs). The thermal spike refers to the formation of a high temperature region around the ion trajectory that can, even, lead to melting. The concept was developed in detail by Toulemonde and co-authors [15-18] to successfully describe the formation of latent amorphous tracks by jon impacts $[19,20]$. However, the thermal spike model alone cannot explain the cumulative character of damage and several features of the structure of the tracks, e.g. the existence of a defective surrounding halo. The exciton-decay model, initially suggested by [toh [21], in combination with the thermal spike concept allows us to calculate the concentration of point defects generated by jon-beam irradiation. Moreover, the model, complemented with a defect-driven 
phase-transformation, also accounts for lattice amorphization. Under a number of simplifying assumptions, it leads to analytical formulae that satisfactorily reproduce a large set of experimental data on $\mathrm{LiNbO}_{3}$. However, such analytical formulation is deterministic and it does not take into account the random statistical nature of jon impacts. Therefore, it does not provide a precise quantification of damage accumulation as a function of ion fluence, particularly at low fluences and stopping powers close to threshold where spatial fluctuations of damage are very relevant.

The goal of this work was to develop a MonteCarlo approach within the excitonic framework to simulate the amount and morphology of swift-ion damage in $\mathrm{LiNbO}_{3}$ as a function of fluence. It considers both the damage generated in over-threshold conditions (amorphous tracks) $[2,22]$ and the damage generated in subthreshold conditions (point defects) [1]. ln addition, the formation of uniform amorphous layers [1,3] at high enough fluence is explained. This occurs either by amorphous track overlapping or by the accumulation of defects generated in sub-threshold conditions that eventually lead to amorphization (cumulative character). Moreover, the kinetics of damage measured by Rutherford Backscattering Spectroscopy in channeling geometry (RBS-C), which yield complex Avrami's type laws [23], can be well reproduced. For future work the statistical nature of the stopping power could also be considered in order to give an even better picture of the injtial structure of the amorphous core tracks. Another advantage of the MonteCarlo approach is the possibility to deal with the synergy between nuclear and electronic damage by combining SRIM and MonteCarlo codes. The formalism is, in principle, applicable to other materials besides $\mathrm{LiNbO}_{3}$.

In Section 1 we will briefly summarize the exciton model. Then, the MonteCarlo approach will be described and finally the morphological and kinetical evolutions will be discussed and compared to experiments.

\section{Non-radiative exciton-decay model}

The model assumes that point defects, possibly consisting of broken or distorted, $\mathrm{Nb}-\mathrm{O}_{6}$ octahedra [24], are formed by nonradiative decay of localized excitons. This decay channel competes with other radiative (and maybe non-radiative) channels. The competition between the two channels is schematically described by a simple energy level diagram shown in Fig. 1. It is essentially similar to that discussed for color center formation in alkali halides $[25,26]$. The excited state presents a certain energy barrier $(\varepsilon)$ that separates the bound and unbound (dissociative) regions in the configuration coordinate $(Q)$. The excitons are formed at a final thermalization state of the electron and hole carriers created by the electronic excitation associated to the electronic stopping power,

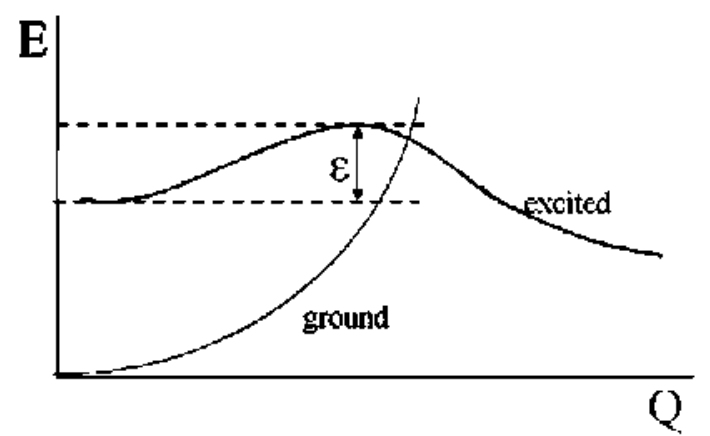

Fig. 1. Schematic diagram showing the energy $E$ for the ground and excited electronic states of a localized or self-trapped exciton as a function of a configurational coordinate $\mathrm{Q}$. Note the energy barrier $\varepsilon$ for non-radiative decay giving rise to defect formation.
$S_{\mathrm{e} .}$ In brief, the processes following ion impact are as follows. First, electrons and holes are created through Coulomb scattering from the incoming ion with the electrons of the material. The electrons, after a short time, present an energy and radial distribution obtained by Waligorski et al. [27] that extends a few nanometers from the jon trajectory. Then, they undergo collisions with other electrons to reach thermalization at an effective temperature, $T_{\mathrm{e}}$. After this stage, electron-phonon interactions come into play to attain a common electron-lattice temperature, $T_{0}$. The process can be described by a set of two coupled rate equations [2] and leads to a roughly gaussian radial distribution with a width, $a$, of around $4.5 \mathrm{~nm}$. This thermal profile will be taken as the starting point $(t=0)$ for our model that will follow the approximate analytical treatment by Szenes [18]. Simultaneously and given the high excitation density, one may consider that all electrons and holes have been coupled into pairs (excitons) with a concentration $N_{X}$ and that they are localized (or self-trapped) in the crystal lattice. From that moment on $(t>0)$, the phonon system cools down and the excitons decay (recombine). In cylindrical coordinates $(r, z), z$ being the track axis, the overall rate of exciton-decay including the defectgeneration and radiative decay channels is [14]

$\frac{d N_{X}}{d t}=-N_{X}(r, z, t)\left[v_{0} \exp \left(-\frac{\varepsilon}{k_{B} T(r, z, t)}\right)+\frac{1}{\tau}\right]$,

where, $v_{0}$ is a frequency factor, $\tau$ the radiative lifetime and $T(z, r, t)$ stands for the evolution of the temperature profile in the spike. Focusing on the defect-generation channel of Eq. (1) one can write the rate of defect-generation as

$\frac{d n}{d t}=N_{X}(r, z, \mathfrak{t}) v_{0} \exp \left(-\frac{\varepsilon}{k_{B} T(r, z, t)}\right)$.

After time integration one obtains for the overall radial distribution of the density of defects,

$n(r, z)=\int_{0}^{\infty} d t v_{0} N_{X}(r, z, t) \exp \left(-\frac{\varepsilon}{k_{B} T(r, z, t)}\right)$

that is illustrated in Fig. 2 for two input stopping powers.

Once we have the expression to calculate the defect concentration profile the model goes a step further. It assumes that when that concentration overcomes a certain threshold value, $n_{\mathrm{th}}$, (see Fig. 2), a defect-driven phase-transformation into an amorphous phase is triggered $[1,14,28]$. Consequently, the region of the track that fulfills that condition corresponds to the so-called amorphous track core. Outside that region the track contains an insufficient defect concentration to induce amorphization (track halo). The

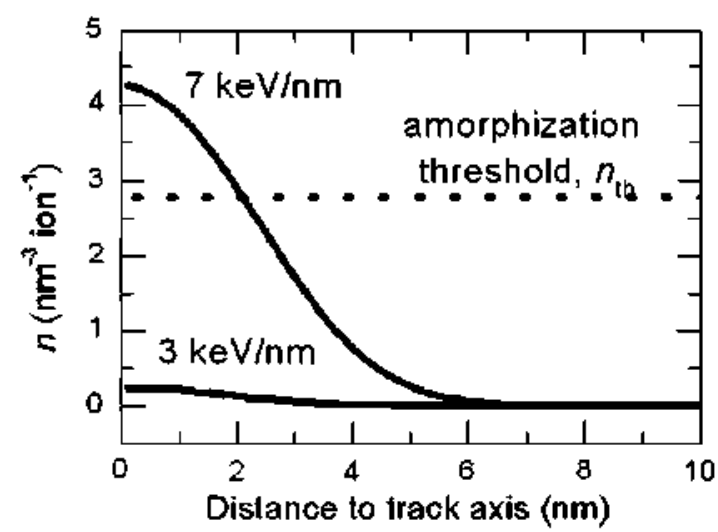

Fig. 2. Defect concentration obtained from the excitonic model for ions with $S_{e}=3$ and $7 \mathrm{keV} / \mathrm{nm}$ impinging on a $\mathrm{LiNbO}_{3}$ crystal. The amorphization threshold $\left(n_{\mathrm{ch}}\right)$ is indicated and corresponds to the defect concentration generated at the surface by an ion with $S_{e}=6 \mathrm{keV} / \mathrm{nm}$. 
condition, $n=n_{\text {th }}$, defines the threshold stopping power $\left(S_{\mathrm{th}}\right)$ required to reach amorphization (thresholding). In the standard thermal spike model this transformation is considered to be melting, but our condition is more general.

\section{MonteCarlo approach}

As it was described in the previous section, the excitonic model provides the defect concentration $n(r, z)$ originated by a single ion. In order to account for the damage evolution in a multiple track scenario we have developed a MonteCarlo algorithm that operates as follows (Fig. 3 is a schematic illustration):

- The sample is divided in thin slabs, typically $50-100 \mathrm{~nm}$ thick, parallel to the surface. Each slab starts at a depth $z$ from the surface.

- Each slab consists of a grid of $100 \times 100$ cells. Each cell has an area (parallel to the surface) of $1 \mathrm{~nm}^{2}$.

- Ions are assumed to hit the sample perpendicular to the surface at a random position $X, Y(0<X, Y<100 \mathrm{~nm})$, see red line in Fig. 3.

- Each ion impinging the sample corresponds to a fluence of $10^{-4} \mathrm{~nm}^{-2}=10^{10} \mathrm{~cm}^{-2}$

- The distance from the center of every cell to the track axis is evaluated in every slab as indicated by the yellow lines in Fig. 3.

- The effect of the ion passage to every cell of the sample is given by the defect concentration, $n(r, z)$, calculated by the excitonic model (Fig. 2). Now $r$, represents the distance of every cell to the track axis. Since the electronic damage is cumulative, the damage generated by the incoming ion in every cell is added up to the damage generated by previous ions.

- If the critical defect concentration $n_{\text {th }}$ is exceeded, we assume that the cell becomes amorphous and thus, its defect concentra-

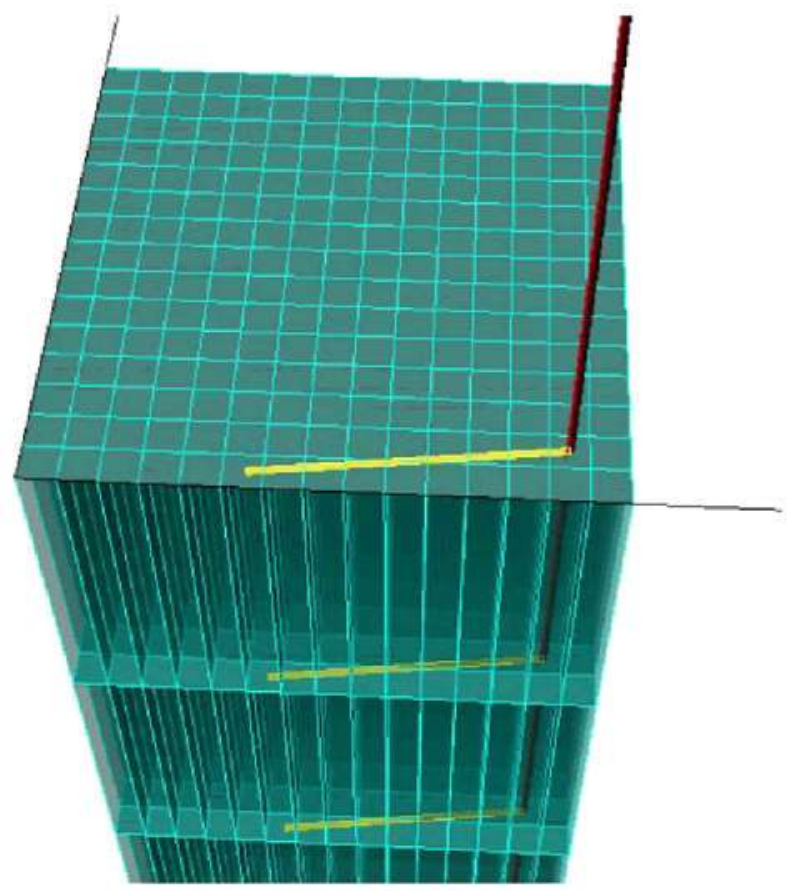

Fig. 3. Schematic illustration of the structure employed for the MonteCarlo algorithm. Note that the sample is divided in slabs parallel to the surface and each slab contains 10,000 identical cells whose area parallel to the surface is $1 \mathrm{~nm}^{2}$. The red line represents an ion trajectory. In order to consider the damage generated by every ion in every cell, the distance to the ion trajectory axis is calculated as it is indicated with the yellow lines for every cell. (For interpretation of the references in colour in this figure legend, the reader is referred to the web version of this article.) tion equals the molecular density of the sample $(N)$. The critical defect concentration, $n_{\text {th }}$, corresponds to the damage concentration generated at the surface by a single ion with stopping power $S_{\text {th. }}$

This procedure provides the defect concentration at any given cell as a function of ion fluence. In addition, it predicts the appearance of amorphous regions not only as an effect of an ion passage with $S_{\mathrm{e}}>S_{\text {th }}$ but as an effect of damage accumulation produced by several ions.

\section{Evolution of damage morphology with stopping power and ion fluence: MonteCarlo simulations}

The MonteCarlo approach allows us to build two-dimensional maps where the local damage level and the morphology of amorphized areas at a given depth from the crystal surface are easily visualized. With regard to stopping power one can consider two cases.

\subsection{Stopping power at the map plane is above threshold}

As an example, defect concentration maps of the surface of a $\mathrm{LiNbO}_{3}$ crystal irradiated with $\mathrm{Cl}$ ions at $45.8 \mathrm{MeV}\left(S_{\mathrm{e}}=6.8 \mathrm{keV} /\right.$ $\mathrm{nm}$ at the surface) are given in Fig. 4 (left) for different fluences. Based on previous works [1] we take as threshold stopping power $S_{\text {th }}=6 \mathrm{keV} / \mathrm{nm}$. For the lowest fluence only one impact (corresponding to a fluence of $10^{10} \mathrm{~cm}^{-2}$ ) is registered. Since $S_{\mathrm{e}}>S_{\mathrm{th}}$. the ion impact produces a single amorphous track (white area) that is surrounded by a colored halo containing point defects whose concentration is below the critical value $n_{\mathrm{th}}$. On increasing fluence more tracks appear and the extension and morphology of the amorphous region is markedly modified. At low fluences the amorphous regions present a cylindrical symmetry surrounded by a defective region (colored area) because they stem from one single ion impact. As the fluence increases, track overlapping takes place and the regular cylindrical shape of the amorphous regions becomes more blurry. Note that the overlap of regions with certain concentration of defects leads to amorphization. This clearly illustrates the cumulative character of the electronic damage. The resulting amorphous fraction for the surface layer is $0.1 \%, 15.4 \%$ and $80.2 \%$ for the fluences $10^{10}, 10^{12}$ and $5 \times 10^{12} \mathrm{~cm}^{-2}$, respectively. The MonteCarlo simulations also predict the depth of the amorphous tracks, Fig. 4 (right). At a fluence of $1 \times 10^{12} \mathrm{~cm}^{-2}$ the amorphous tracks reach a depth of around $6 \mu \mathrm{m}$. In overlapping track regime $\left(5 \times 10^{12} \mathrm{~cm}^{-2}\right)$, one observes that the amorphous depth increases approaching $7 \mu \mathrm{m}$. It is the cumulative character of the electronic damage that makes both the amorphous depth (right) and the amorphous area (left) increase with fluence.

\subsection{Stopping power below threshold}

Fig. 5 (left) illustrates defect concentration maps at the surface of a $\mathrm{LiNbO}_{3}$ crystal irradiated with $\mathrm{Si}$ ions at $5 \mathrm{MeV}\left(S_{\mathrm{e}}=4.2 \mathrm{keV} /\right.$ $\mathrm{nm}$ at the surface). In this case, single ions do not form amorphous tracks but only defective spots, as observed in Fig. 5a corresponding to a fluence of $10^{10} \mathrm{~cm}^{-2}$ (only one impact). The amorphous regions appear as a consequence of defect accumulation after every ion passage. At a fluence of $5 \times 10^{12} \mathrm{~cm}^{-2}$, some amorphous regions start to develop. They do not present cylindrical symmetry because they occur as a result of multiple track overlapping. This is more evident at higher fluence. At $10^{13} \mathrm{~cm}^{-2}$, the amorphous fraction amounts to $20.6 \%$ and the shape of amorphous regions do not resemble a set of single ion tracks at all. One sees from the sequence $5 \mathrm{a} \rightarrow 5 \mathrm{~b} \rightarrow 5 \mathrm{c}$, that for $S_{\mathrm{e}}<S_{\text {th }}$ a certain minimum flu- 

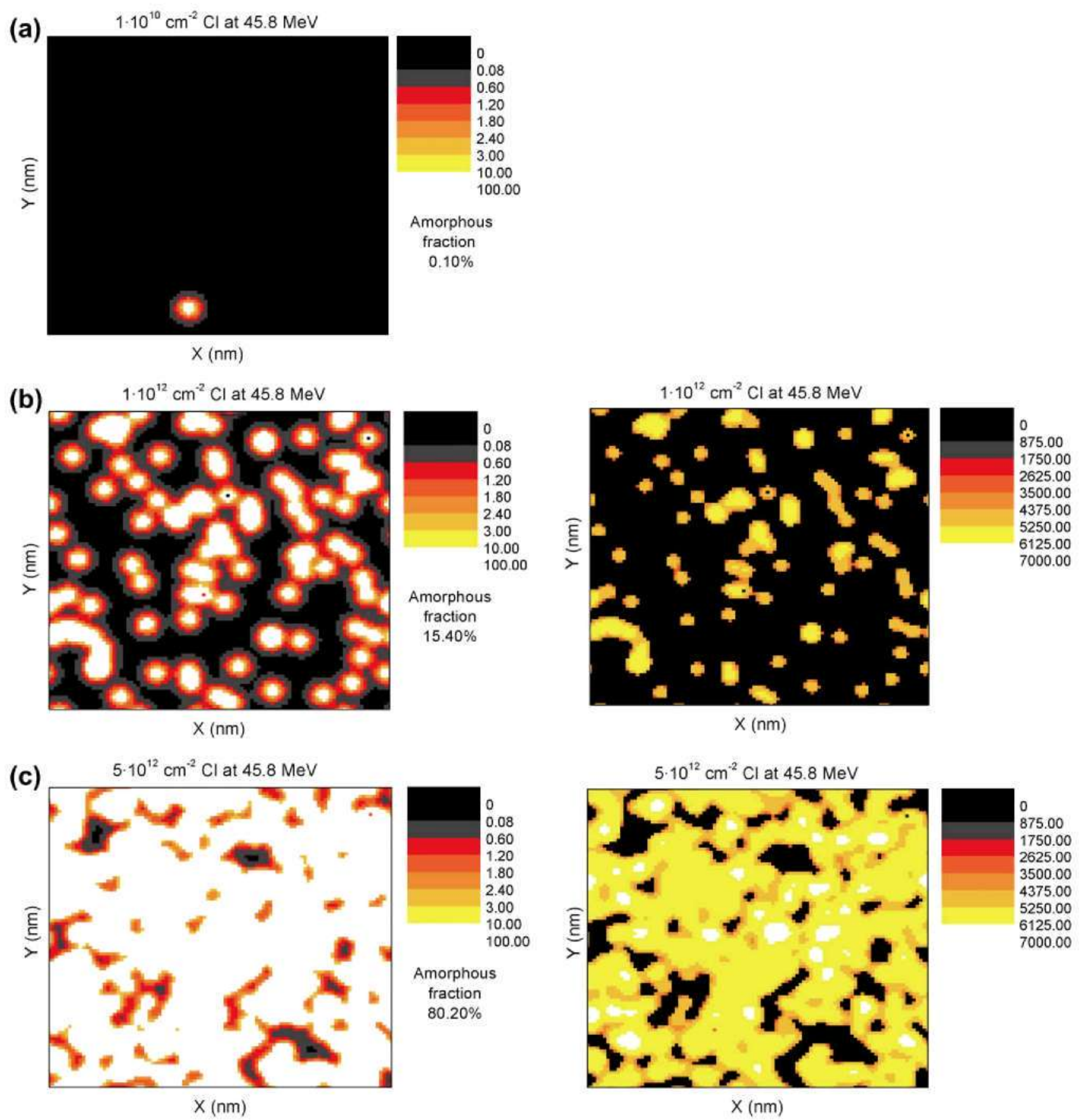

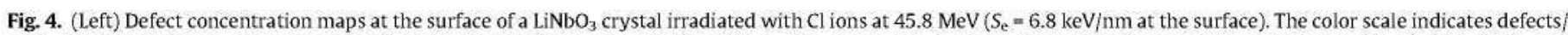

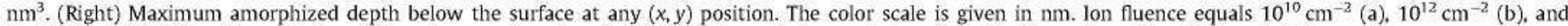
$5 \times 10^{12} \mathrm{~cm}^{-2}$ (c). (For interpretation of the references in colour in this figure legend, the reader is referred to the web version of this article.)

ence is needed before amorphization starts (incubation fluence). Note that the amorphous depth (right) is now very small $(<300 \mathrm{~nm})$. The role of defect-generation is important in this case. On the other hand, when $S_{\mathrm{e}}>S_{\mathrm{th}}$, every impinging ion generates an amorphous track. Then, the role of the amorphous core of the track is dominant from the point of view of sample properties or defect analysis, while the surrounding defective regions play a minor role, as will be discussed below.

Appropriate experiments to observe the morphology patterns predicted by our MonteCarlo approach are difficult. A possibility would be to use high resolution SNOM that would easily distinguish the crystalline and amorphous regions of the pattern due to the very different refractive indices $\left(n_{a}=2.10\right.$ for the amorphous phase, and $n_{o}=2.28$ and $n_{e}=2.20$ for the crystalline phase). However, the attainable resolution $(\sim 50 \mathrm{~nm})$ is still insufficient for adequate observation. Alternatively, one may appreciate that the patterns resemble those achieved after suitable selective chemical etching treatments [29] that excavate the amorphous regions of the surface while leaving essentially undisturbed the crystalline zones. Although the comparison is suggestive, the etching is an aggressive technique that strongly modifies and broadens the features initially present in the irradiated surface.

However, one can focus on a particular relevant feature of the simulations. For irradiations below threshold and low enough flu- 

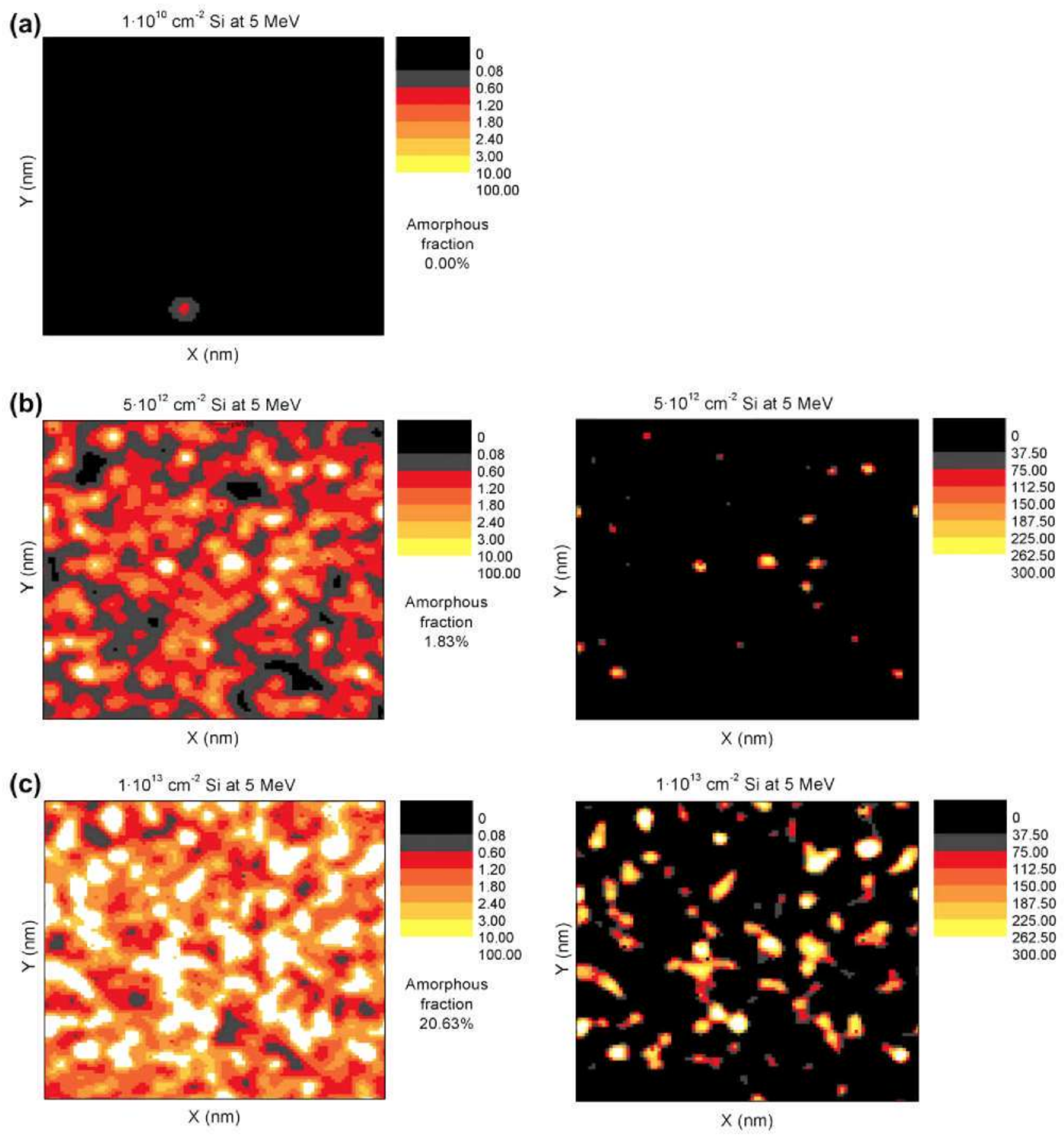

Fig. 5. (Left) Defect concentration maps at the surface of LiNbO $\mathrm{C}_{3}$ crystal irradiated with $\mathrm{Si}$ ions at $5 \mathrm{MeV}\left(\mathrm{S}_{\mathrm{e}}=4.2 \mathrm{keV} / \mathrm{nm}\right.$ at the surface). The color scale indicates defects/nm ${ }^{3}$. (Right) Maximum amorphized depth below the surface at any $(x, y)$ position. The color scale is given in nm. Ion fluence equals $10^{10} \mathrm{~cm}^{-2}(\mathrm{a}), 5 \times 10^{12} \mathrm{~cm}^{-2}(\mathrm{~b}), \mathrm{and}_{10} 0^{13} \mathrm{~cm}^{-2}$ (c). (For interpretation of the references in colour in this figure legend, the reader is referred to the web version of this article.)

ences, amorphous spots are not generated as observed in Fig. 5a. A certain incubation fluence is needed to make them appear as a consequence of track overlapping. This incubation fluence to induce amorphization has been, indeed, confirmed [23] by data of damage kinetics obtained by means of RBS-C. A detailed discussion of this point will be addressed in the next section.

\section{Kinetics of damage}

The described MonteCarlo approach is suitable to obtain the kinetics for the two components of damage, namely amorphized and disordered (non amorphous) regions. These two cases will be now discussed.

\subsection{Kinetics of amorphization}

From the defect concentration maps one can plot the quantitative evolution of the amorphous fraction for every slab as a function of fluence for a given incident ion. Fig. 6 shows the amorphous fraction of the surface layer of a $\mathrm{LiNbO}_{3}$ crystal as a function of fluence for several ions calculated from the model. They present an S-shape typical of Avrami-type kinetics. Note that the higher the stopping power the lower the fluence required for full 


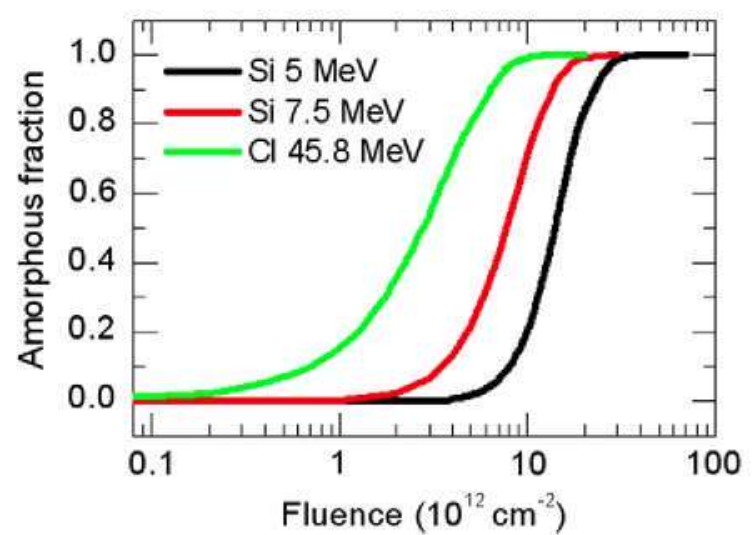

Fig. 6. Amorphous fraction at the surface of a $\mathrm{LiNbO}_{3}$ crystal irradiated with $5 \mathrm{MeV}$ Si ions $\left(S_{\mathrm{e}}=4.2 \mathrm{keV} / \mathrm{nm}\right), 7.5 \mathrm{MeV} \mathrm{Si}$ ions $\left(S_{\mathrm{e}}=4.9 \mathrm{keV} / \mathrm{nm}\right)$ and $45.8 \mathrm{MeV} \mathrm{Cl}$ ions $\left(S_{\mathrm{e}}=6.8 \mathrm{keV} / \mathrm{nm}\right)$. The amorphous fraction is obtained from the MonteCarlo algorithm and given as a function of the ion irradiation fluence.

amorphization. In addition, it is very interesting to note that the shape of the curve corresponding to irradiations with $45.8 \mathrm{MeV}$ $\mathrm{Cl}$ ions differs with respect to the other two curves. The reason is that the latter curves correspond to irradiations in sub-threshold conditions, i.e., an incubation fluence is needed to produce amorphous phases. In contrast, when irradiations are performed with $45.8 \mathrm{MeV} \mathrm{Cl}$ ions every single ion is able to produce an amorphous track. In other words, every single ion contributes directly to increase the amorphous fraction. The features observed in Fig. 6 are essentially in agreement with the results of detailed RBS-C experiments. However, one has to be aware that this technique measures disorder and not only amorphization. Therefore, for a meaningful comparison between theory and experiment one has to carefully discuss the information gathered in the RBS-C experiments.

\subsection{Kinetics of disorder: RBS-C experiments}

Most data on the evolution of damage with ion-beam fluence are derived from RBS-C experiments. In them, samples are probed with a hydrogen or helium beam aligned along a crystallographic axis or planar direction, and the reduction in RBS yield in comparison to that obtained under random incidence is measured. The problem with the technique is that it does not distinguish between fully amorphous or defective areas, so it yields information on the disorder. In order to properly analyze the data one should introduce a filter function as in Ref. [30]. A suitable filter function would require a detailed knowledge of the backscattering yield of each of the point defects generated by irradiation. This problem is not simple. A reasonable and useful approximation for $\mathrm{LiNbO}_{3}$ considers that the yield corresponds to an atomic $(\mathrm{Nb})$ size cross-section, i.e., a filter function is introduced:

$n^{*}=f n \quad$ for $n<n_{\text {th }}$

$n^{*}=N \quad$ for $n \geqslant n_{\text {th }}$

where $n^{*}$ is the effective concentration of backscattering centers, $n$ is the real concentration of defects generated by irradiation, $f$ is the backscattering factor, which represents the enhancement/reduction factor in the scattering cross-section for each individual point defect in comparison to the area of the atomic cell, $N$ is the density of atoms in the sublattice mostly contributing to RBS-C, i.e., $18.9 \mathrm{Nb}$ atoms $\mathrm{nm}^{-3}$ for $\mathrm{LiNbO}_{3}$. When $n>n_{\text {th }}$, the whole volume becomes amorphized and contributes to random scattering. The filter function is plotted in Fig. 7. The use of the filter function allows one to compare the experimental de-channeled fraction obtained by RBS-C with the results of our model. For every possible value of $f$,

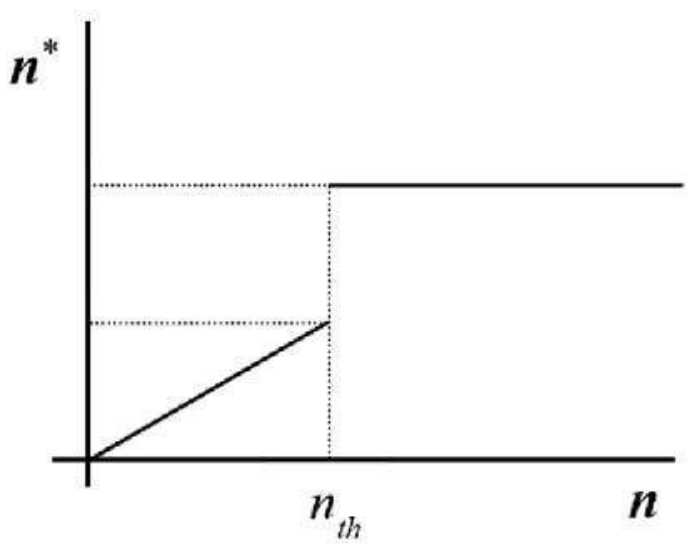

Fig. 7. Filter function for the relation between the effective concentration of backscattering centers $\left(n^{*}\right)$ and the concentration of defects generated by irradiation $(n)$.
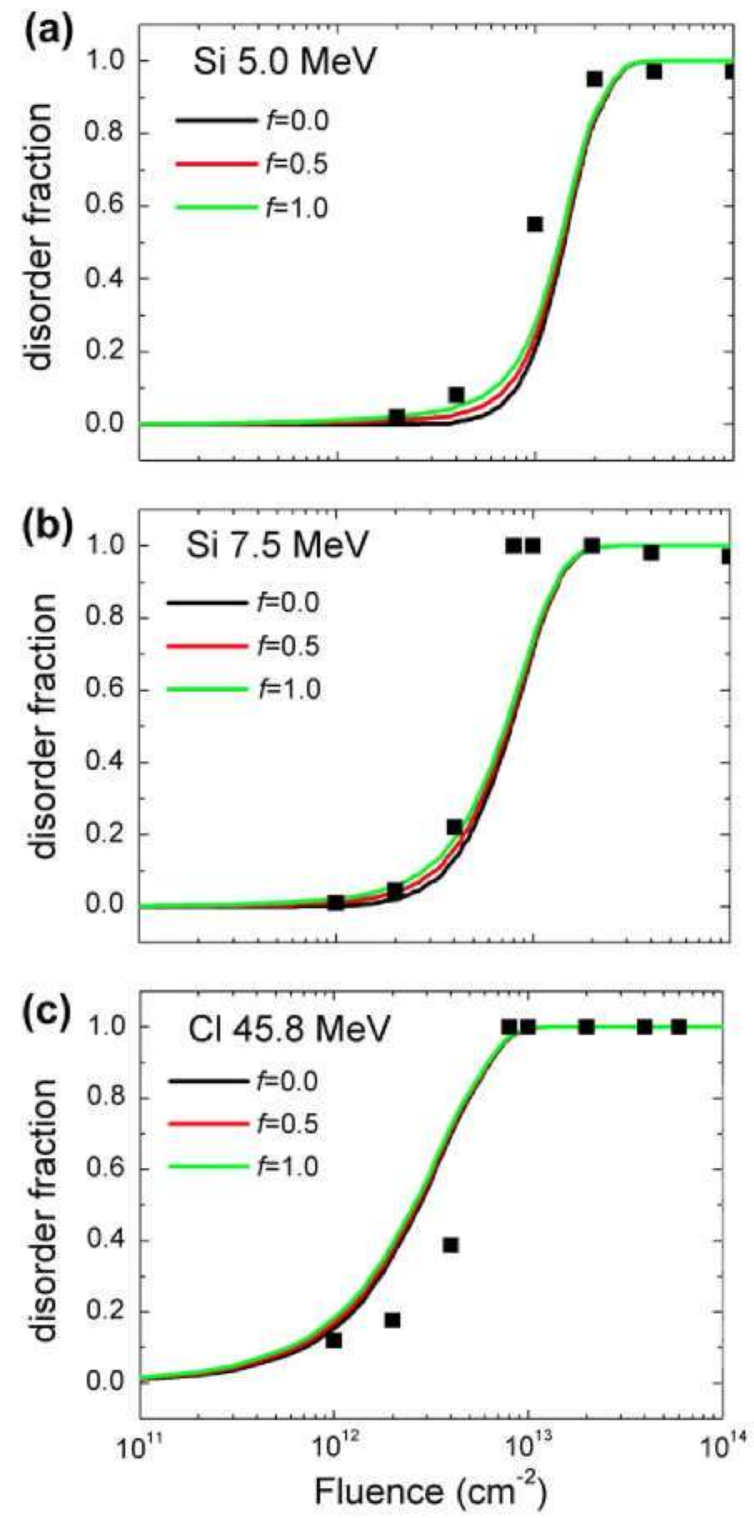

Fig. 8. Disordered surface fraction obtained from RBS-C as calculated from the model for different values of the backscattering factor, $f, \mathrm{LiNbO}_{3}$ has been irradiated with (a) $5 \mathrm{MeV}$ Si ions, (b) with $7.5 \mathrm{MeV} \mathrm{Si}$ ions, and (c) with $45.8 \mathrm{MeV} \mathrm{Cl}$ ions. 
we can get the de-channeled fraction that RBS-C would provide as $n^{*} / N$.

Fig. 8 shows disordered areal fractions obtained by RBS-C and by the exciton model for different values of the backscattering factor, $f$. The results obtained by the model reproduce well the experimental values. Note that phenomena like the so-called "velocity effect" [31,32] are not included in the model and may play an important role. The curves obtained for $f=0$ are identical to those presented in Fig. 6 because $f=0$ implies that only amorphization is considered and the defective regions produced by irradiation are neglected. From Fig. 8a and b it is clear that neglecting the defective regions leads to a bad agreement with the experimental results. On the other hand, the curves obtained from the model for various values of $f$ in the case of $45.8 \mathrm{MeV} \mathrm{Cl}$ irradiation (Fig. $8 \mathrm{c}$ ) are very close to each other. The reason is that such an irradiation leads to the production of single amorphous tracks by every single ion, which become dominant over the defective halos. This fact evidences that in the presence of amorphous regions the surrounding defective regions play a minor role.

\section{Generation of uniform amorphous layers: some statistical features}

Our MonteCarlo model predicts full (100\%) amorphization of a surface for stopping powers above threshold and high enough fluences (see saturation stage in Figs. 6 and 8). Moreover, all slabs of the crystal subjected to those conditions become amorphous so that, an homogeneous amorphous layer is produced by the irradiation. To deal with this situation an analytical formulation of the
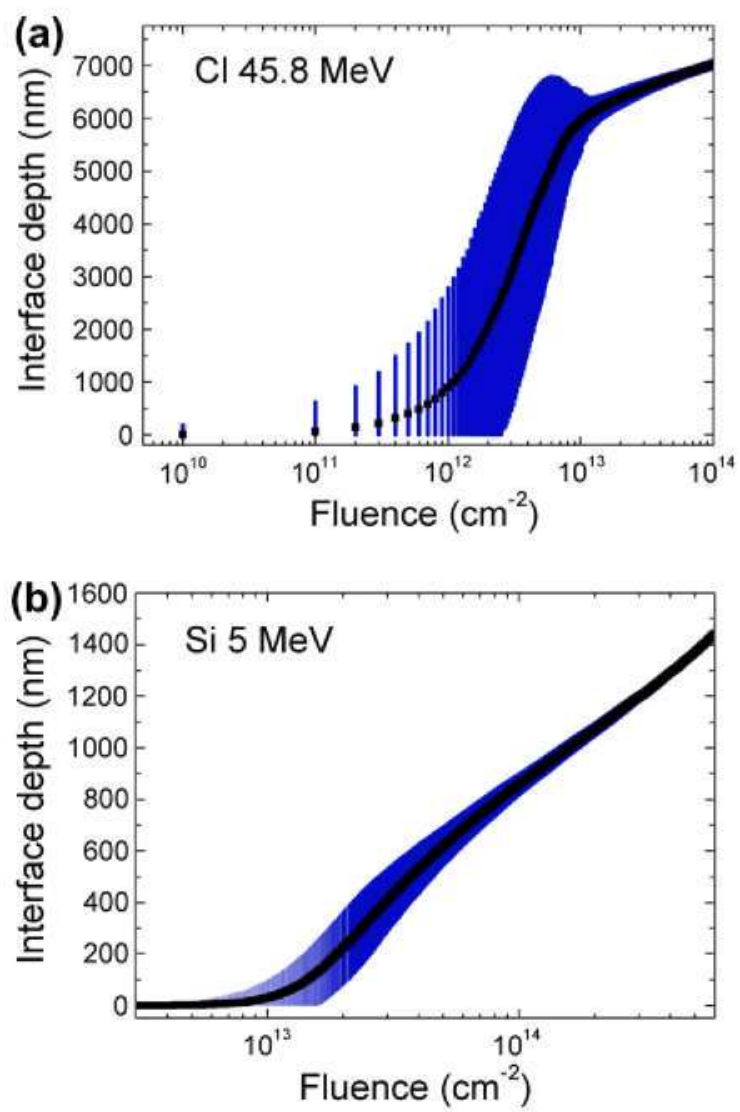

Fig. 9. Average amorphous depth ( $\mathrm{nm}$ ) as a function of fluence. This value gives an idea about the evolution of the amorphous region as a function of fluence. The error bars represent the standard deviation and they are an estimation of the amorphouscrystalline interface roughness. (a) The crystal is irradiated with $\mathrm{Cl}$ at $45.8 \mathrm{MeV}$, and (b) the crystal is irradiated with $\mathrm{Si}$ at $5 \mathrm{MeV}$ exciton model that ignores statistical aspects is appropriate and has been developed in [14]. The model predicts that the thickness of the homogeneous amorphous layer increases appreciably with fluence, i.e. the crystalline-amorphous interface propagates into the crystal. The amorphous thickness as a function of fluence was satisfactorily described within the analytical model for the case of $\mathrm{LiNbO}_{3}$. In fact, the tailoring of these layers provides a useful technique to fabricate optical waveguides as an advantageous alternative to the standard ion implantation technique [33]. Then, which are the advantages of a MonteCarlo vs. an analytical approach in relation to those amorphous thick layers? The MonteCarlo approach is the only suitable tool to deal with several relevant features that are associated to the statistical nature of the ion-matter interaction. There is one particular aspect that may play a relevant role for photonic (waveguide) applications, namely the definition (boundary) of the amorphous-crystalline interface. In fact, the MonteCarlo method provides a unique way to simulate the microstructure of the interface, i.e. its roughness and abruptness. Let us compare the situation for irradiations with $\mathrm{Si}$ at $5 \mathrm{MeV}$ and $\mathrm{Cl}$ at $45.8 \mathrm{MeV}$. From the MonteCarlo results we can extract the local amorphous depth at any $(x, y)$ position for every fluence as shown in the right side of Figs. 4 and 5. Averaging the local amorphous depth to all the $(x, y)$ positions (10,000 cells), we get the average amorphous depth together with its standard deviation. In Fig. 9 we plot the average amorphous depth for the two considered irradiation cases as a function of fluence using the standard deviation as error bars. Note that the homogeneous amorphous depth obtained from the analytical model gives the thickness of fully amorphous uniform layers. The average amorphous depth calculated now with the MonteCarlo formalism does

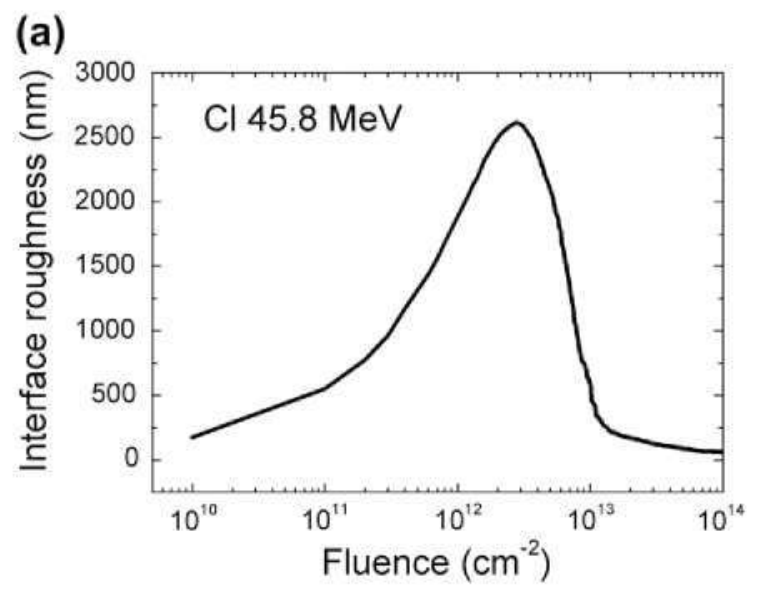

(b)

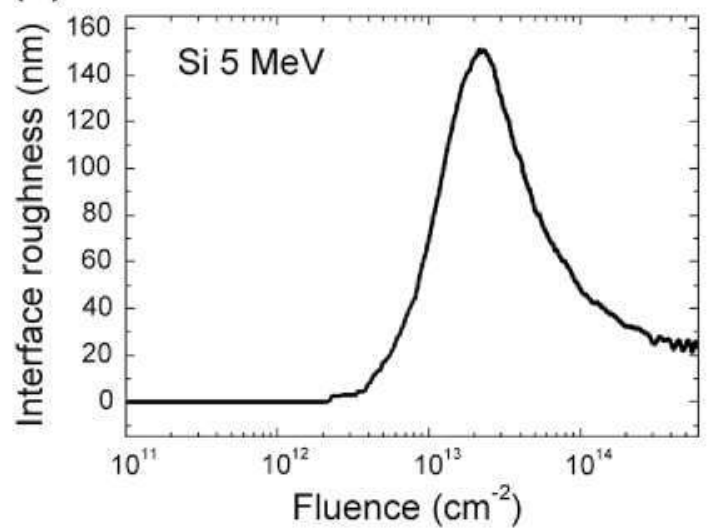

Fig. 10. Roughness of the crystalline-amorphous interface as a function of fluence for irradiations with (a) $\mathrm{Cl}$ ions at $45.8 \mathrm{MeV}$, and (b) Si ions at $5 \mathrm{MeV}$. 
not consider whether a fully amorphous layer is formed or not, it simply gives an idea about the depth evolution of the amorphous-crystalline interface as a function of depth. Moreover, one can use the standard deviation of the average amorphous depth as a parameter of roughness. In other words, it is an useful estimation of the width of the crystalline-amorphous boundary. In Fig. 10 it is plotted for irradiations with both jons. One should note that the coexistence of crystalline regions within the amorphous layer has been confirmed by micro-Raman spectroscopy [3,33]. As expected, one sees that the boundary is much better defined for high fluences and that the definition is bad for low fluences where the layer is not yet truly amorphous. The importance of these results for the optimization of waveguide performance (e.g. losses) appears very relevant.

\section{Summary and conclusions}

A MonteCarlo approach to the non-radiative exciton-decay model has been developed. It takes into account the statistical spatial distribution of the jon impacts at any depth in the sample (in particular, at the sample surface). At variance with the previous analytical formulation, the new method is able to describe adequately the range of low irradiation fluences where overlapping is still poor and statistical divergences of the true amorphized area with regard to the average values are quite significant. A good representation of the Avrami's type kinetics for amorphization has been achieved. On the other hand, the thickness of the layers having a mixed amorphous-crystalline composition has been obtained as a function of fluence. We consider that the MonteCarlo approach will be a useful tool to deal with other pending jon-beam damage problems such as the coupling between the disorders caused by nuclear elastic collisions and electronic excitations.

\section{Acknowledgements}

We acknowledge financial support from the Spanish Ministery of Science and lnnovation for the projects MAT-2005-06359-CO302 and MAT-2008-06794-C03-03. A. Rivera thanks the 13P program of CSIC for financial support.

\section{References}

[1] A. Rivera, J. Olivares, G. García, J.M. Cabrera, F. Agulló-Rueda, F. Agulló-López, Plys. Status Solidi A 206 (6) (2009) 1109

[2] M. Toulemonde, W. Assman, C. Dufour, A. Meftah, F. Studer, C. Trautmann, in: P. Sigmund (Ed.), lon Beam Science: Solved and Unsolved Problems, The Royal Danish Academy of Sciences and letters, Copenhagen, 1996, p. 263.
|3| J. Olivares, G. García, F. Agulló-López, F. Agulló-Rueda, A. Kling. J.C. Soares. Appl. Phys. A 81 (2005) 1465.

|4| G.G. Bentini, M. Bianconi, L. Correra, M. Chiarini, P. Mazzoldi, C. Sada, N. Argiolas, M. Bazzan, R. Guzzi, ]. Appl. Phys. 96 (2004) 242.

[5] A. García-Navarro, J. Olivares, G. García, F. Agulló-López, S. García-Blanco, C Merchant, J. Stewart Aitchison, Nucl. Jnstrum. Meth. Phys. Res, B 249 (2006) 177.

|6] J. Jensen, M. Supinski, K. Hjort, R. Sanz, Nucl. Instrum. Metlı. Plyss, Res. B 266 (2008) 3113.

|7| A. Kamarou, W. Wesch, E. Wendler, A. Undisz. M. Rettenmayr, Phys. Rev. B 73 (2007) 184107.

[8] CS. Schnor, P. Kluth, A.P. Byme, C.J. Foran, M.C. Ridway, Phys. Rev. B 77 (2008) 073204.

|이 J. Olivares, A García-Navarro, G. García, F, Agulló-lópez, F. Agulló-Rueda, A García-Cabanes, M. Carrascosa, J. Appl. Phys. 101 (2007) 33512

[10] J.F. Ziegler, J.P. Biersack, U. Littmark, The Stopping and Ranges of lons in Solids, Pergamon Press, New York, 1985, see also the SRIM webpage, <http:f www.sim.olgl>

[11] J.F. Ziegler, lon Implantation Technology, North Holland, Amsterdam, 1992.

[12] P.D. Townsend, P.j. Chandler, L. Zhang, Optical Effects of Ion Implantation, Cambridge University Press, Cambridge, 1994

[13] F. Agulló-López, A. Méndez, G. García, J. Olivares, J.M. Cabrera, Phys. Rev. B 74 (2006) 174109 .

[14] A. Rivera, A. Méndez, G. García, J. Olivares, J.M. Cabrera, F. Agulló-lópez, J. Lumin. 128 (2008) 703.

[15] M. Toulemonde, Ch. Dufour, A. Meftah, E. Paumier, Nucl, Instrum. Metl. Phys. Res. B $166\lceil 167(2000) 903$.

[16] M. Toulemonde, C. Trautmann, E. Balanzat, K. Hjort, A. Weidinger, Nucl Instrum. Meth. Phys. Res. B 1 (2004) 216.

[17] A. Meftah, J.M. Costantini, N. Khalfaoui, S. Boudjadar, J.P. Stoquert, F. Studer, M Toulemonde. Nucl. Instrum. Meth. Plys. Res. B 237 (2005) 563.

[18] G. Szenes, Phys. Rev. B 60 (1999) 3140.

[19] R.l. Fleischer, P.B. Price, R.M. Walker, Nuclear Tracks in Solids. Principles and Applications, University of California Press, Berkeley, 1975.

[20] R. Spohr, in: K. Bethge (Ed.), lon Tracks and Microtechnology: Basic Principles and Applications, Vieweg. Braunschweig. 1990.

[21] N. Jtoh, Nucl. Instrum. Meth. Phys. Res. B 135 (1998) 175.

[22] B. Canut, S.M.M. Ramos, R. Brenier, P. Thevenard, J.L. Loubet, M. Toulemonde, Nucl. Jnstrum. Meth. Phys. Res. B 107 (1996) 194.

[23] A. García-Navarro, M. Bianconi, J. Olivares, F. Agulló-López, G. García, J. Appl. Phys. 101 (2007) 83506.

[24] G. García, J. Olivares, F. Agulló-López, A. García-Navarro, F. Agulló-Rueda, A. García-Cabañes, M. Carrascosa, Europhys. Lett. 76 (2006) 1123.

[25] F. Agulló-López, R.C. Catlow, P.D. Townsend, Point Defects in Materials. Academic Press, london, 1984.

[26] N. Itol,. A.M. Stoneham, Materials Modification by Electronic Excitation, Cambridge University Press, 2001.

[27] M.P.R. Waligorski, R.N. Hamm, R. Katz, Nucl. Tracks Radiat. Meas. 11 (1986) 309

[28] H.]. Fecht, Nature 356 (1992) 133

[29] A. García-Navarro, A. Méndez, J. Olivares, G. García, F. Agulló-López, M. Zayat. D. levy. L Vazquez. Nucl. Instrum. Meth. Plyys. Res. B 249 (2006) 172.

[30] A. Rivera, J. Olivares, M.L. Crespillo, G. García, M. Bianconi, F. Agulló-López, Nucl, Instrum. Meth. Phys. Res. B 267 (2009) 1460.

[31] A. Meftah, F. Brisard, J.M. Constantini, M. Hage-Ali, J.P. Stoquert, F. Studer, M. Toulemonde, Phys. Rev. B 48 (1993) 920.

[32] G. Szenes, Plyys. Rev, 52 (1995) 6154

[33] J. Olivares, A. García-Navarro, G. García, F. Agulló-López, F. Agulló-Rueda, A García-Cabaĩes, M. Calrascosa, J. Appl, Phys. 101 (2007) 033512. 